to a wide spectrum of viral and bacterial diseases, and might include vaccines, immune globulins, antibiotics, and antiviral medications. All health-care institutions should ensure to have systems in place to facilitate postexposure assessment, and have prophylaxis readily accessible for timely administration. The protocol should describe procedures for the rapid provision of medical care during all work hours (day, evening, and night shifts). Postexposure management approach will depend on the type and extent of exposure, characteristics of the infectious agent (eg, virulence, infectious dose), status of the source patient, the exposed person's susceptibility to infectious diseases of concern, and the relative risks and benefits of the PEP regimen in each individual situation. Occupational exposures should be considered urgent medical concerns to ensure timely postexposure management. Exposed HCP should be monitored for signs and symptoms of infection and for possible adverse effects from drugs. In the absence of PEP, recommendations for postexposure management are intended to achieve early identification of disease and, if present, referral for evaluation of treatment options. Management of exposures should always be fully documented and exposures reported to the appropriate administrative department. A key administrative component is provision of resources for maintenance of infection control and occupational health programs that are responsive to emerging needs.

\section{PREVENTION OF LOW BACK PAIN IN HCWS}

Ruddy Facci*. INSAT - Curitiba, Brazil

10.1136/oemed-2018-ICOHabstracts.944

Round table discussion regarding the evaluation and prevention of Low Back Pain in Health Care Workers.

Presenters: Tello $S^{1}$, Shaw $W^{2}$, Alvarez-Casado $E^{3}$, Facci $\mathrm{R}^{4}$

${ }^{1}$ Centro de Ergonomía Aplicada (CENEA), Barcelona, Spain

${ }^{2}$ University of Massachusetts Medical School, Worcester Massachusetts, USA

${ }^{3}$ Centro de Ergonomía Aplicada (CENEA), Barcelona, Spain

${ }^{4}$ INSAT, Curitiba, Brazil

\section{2b LOW BACK PAIN IN HEALTH CARE WORKERS: A GROWING FOCUS ON SECONDARY PREVENTION}

WS Shaw. University of Massachusetts Medical School, Worcester, Massachusetts, USA

\subsection{6/oemed-2018-ICOHabstracts.945}

Low back pain (LBP) is a ubiquitous problem affecting nearly half of all workers in the health care $(\mathrm{HC})$ setting. Hospital nurses report a 35 percent point prevalence and a 55 percent annual prevalence of LBP. Nursing is among the occupations ranked highest in work-related LBP, so substantial research efforts have been dedicated to understanding the interactions between workplace demands and worker characteristics that increase risk of LBP. Patient lifting and transfer is clearly the most prominent causal factor, and usual interventions include lift/transfer devices, no-lift policies, and ergonomic assessments.
Efforts to reduce physical ergonomic exposures in hospitals and nursing homes have met with some success, but engineering and policy solutions are only effective if coupled with sufficient worker training and participation. Besides the high physical exposures in the HC setting, research has also highlighted the importance of organisational and psychosocial factors in LBP incidence rates. These include high psychosocial demands (when paired with low job control), effort-reward imbalance, and low social support. Thus, LBP prevention efforts in the HC setting need to address psychosocial as well as physical job characteristics. Given the high prevalence of LBP among HC workers, one question is whether more efforts should be directed toward secondary prevention focusing on job accommodation, return-to-work facilitation, and other aspects of organisational support to prevent longterm work absence and job loss after the initial report of LBP. Such disability prevention efforts, when instituted at the organisational level, have shown sizable cost benefits to employers and insurers, but their effects on long-term employee health and well-being lack sufficient evidence. These studies do, however, suggest that how HC organisations respond to individual workers with LBP may be equally important as their systemic efforts to reduce LBP incidence rates.

\section{2d WORK CONDITIONS IN BRAZILIAN HOSPITALS REGARDING PREVENTION OF LOW BACK PAIN}

R Facci. INSAT - Curitiba, Brazil

\subsection{6/oemed-2018-ICOHabstracts.946}

Introduction Low back pain is a very common problem among workers in the healthcare sector worldwide.

In the international scientific literature, healthcare workers involved in caring for dependent patients are among those mos prone to acute and chronic musculoskeletal disorders, especially the dorsolumbar spine. According to WHO 2011, there are around 19.300.000 nursing staff members working in healthcare facilities around the globe (85\% are women). Most healthcare workers manually handle patients on a daily basis, activity 'potentially' in terms of workplace prevention, thus hospital management to adopt effective risk assessment, management and containment measures. The large number of nursing staff are affected by musculoskeletal disorders, preventing them fro performing activities for manually handling patients; the level of sick days taken due to musculoskeletal problems is extremely high. These aspects raise inefficiency, generate higher costs and lower the quality of care.

Methods MAPO Methodology (ISO TR 12296) has been used for evaluating the risk of low back pain among healthcare workers in 3 hospitals in Curitiba. One of the characteristic features of the MAPO Method is that it combines all the various risk factors into a single formula. Three hospitals in Curitiba (surgical and orthopaedic sectors) ahs been the target of this study.

Result The risk value in each sector/hospital has been stablished based upon the different patients, according to their diseases and workplace conditions. 